\section{"Nano Gold"}

Meeting

June 30 and July 1 , 2005, campus Boucicaut, Paris

A meeting entitled "Gold nanoparticles: an interdisciplinary approach" took place on the campus Boucicaut of the Universities Paris VI et Paris VII, last June 30 and July 1, 2005. In spite of the informal organisation of this meeting, it managed to gather about 70 French researchers and PhD students. Its goal was to provide a forum for researchers involved in gold nanoparticles, but working in different scientific fields, such as chemistry, physics and biology.

\section{Why organise a meeting on gold nanoparticles?}

- in chemistry, for instance because when gold nanoparticles are dispersed onto oxide supports, they lead to very promising catalysts with even unique properties for some reactions;

- in physics, because their properties of plasmon resonance give them specific optical and electronic properties compared to bulk gold, they may lead to multiple possible applications providing shape, size of nanoparticles can be controlled as well their arrangement on a substrate; this also requires in situ characterisations;

- in biology, because their bio-compatibility and nontoxicity, their easy functionalisation via organic thiol groups makes gold nanoparticles very attractive for applications in imaging as biological tracer, in the field of bio-captors, and more recently in that of therapy.

This meeting was organised by Dr Catherine Louis, physicochemist of the "Laboratoire de Réactivité de Surface", University Paris VI, and Olivier Pluchery, physicist of the "Institut des NanoSciences de Paris" (INSP), Universities Paris 6 et Paris 7. It was structured around four sessions, each session starting by a key-note lecture:

- Physics, fundamental properties and applications;

- Preparation of gold nanoparticles;

- Chemistry, catalysis;

- Biology, bio-captors;

A poster session was also organised at the end of the first day. The meeting ended with a round table gathering around thirty people. They all appreciated the scientific quality level of the communications and this new opportunity for researchers of different scientific fields to meet. Beyond the common interest for gold nanoparticles, these two days showed that there is a true scientific continuum from one domain to another, and that it would very fruitful to continue this type of inter-disciplinary meeting. This meeting has already permitted thorough discussions between researchers of different domains, and some collaboration should start already. The conclusion of the round table was that most of the participants are eager to continue and deepen this type of scientific exchanges, but within a more formal and structured framework, for instance under the auspices of CNRS (French National Center of Scientific Research) within the framework of the so-called GDR (Groupement of Research).

The program and most of the communications can be found (in French) on the web site:

http://www.insp.upmc.fr/ornano/ornano_accueil.htm 